\title{
LINEAR QUANTUM SYSTEM TRANSFER FUNCTION REALIZATION USING STATIC NETWORKS FOR INPUT/OUTPUT PROCESSING AND FEEDBACK*
}

\author{
SYMEON GRIVOPOULOS ${ }^{\dagger}$ AND IAN PETERSEN AN $^{\ddagger}$
}

\begin{abstract}
The realization of the transfer functions of linear quantum stochastic systems (LQSSs) is of fundamental importance for the practical applications of such systems, especially as coherent controllers for other quantum systems. So far, most works that have addressed this problem have used cascade realizations. In this paper, a new method is proposed, where the transfer function of an LQSS is realized by a series connection of two linear static networks, and a reduced LQSS. The introduction of pre- and postprocessing static networks leaves an intermediate reduced LQSS with a simple input/output structure, which is realized by a simple feedback network of single-mode LQSSs. The key mathematical tool that allows for the construction of this realization is an SVD-like decomposition for doubled-up matrices in Krein spaces. Illustrative examples are provided for the theory developed.
\end{abstract}

Key words. control of quantum systems, linear quantum stochastic systems, transfer function realization

AMS subject classification. $81 Q 93$

DOI. $10.1137 / 15 \mathrm{M} 104829 \mathrm{X}$

1. Introduction. Linear quantum stochastic systems (LQSSs) are a class of models that are widely used in quantum optics and other areas [5, 35, 36]. In quantum optics, they describe a variety of devices, such as optical cavities and parametric amplifiers, as well as networks of such devices. The mathematical framework for these models is provided by the theory of quantum Wiener processes and the associated quantum stochastic differential equations [29, 22, 13]. Potential applications of quantum optics include quantum information and photonic signal processing; see, e.g., $[23,15,32,41,39]$. Another particularly important application of LQSSs is as coherent quantum feedback controllers for other quantum systems, i.e., controllers that do not perform any measurement on the controlled quantum system and thus have the potential for increased performance compared to classical controllers; see $[37,38,14,28,20,21,12,2]$.

A problem of fundamental importance for applications of LQSSs is the problem of realization/synthesis: Given an LQSS with specified parameters, how does one engineer that system using basic quantum optical devices, such as optical cavities, parametric amplifiers, phase shifters, beam splitters, and squeezers? The synthesis problem comes in two varieties. First, there is the strict realization problem which we just described. This type of realization is necessary in the case where the states of the quantum system are meaningful to the application at hand. Examples include

\footnotetext{
${ }^{*}$ Received by the editors November 13, 2015; accepted for publication (in revised form) September 5, 2017; published electronically October 24, 2017. The main results of this work, Theorems 3.1, 4.1, and 4.9, were presented at the 55th IEEE Conference on Decision and Control, Las Vegas, NV, $2016[11]$.

http://www.siam.org/journals/sicon/55-5/M104829.html

Funding: This research was supported by the Australian Research Council under grant FL110100020 and by the Air Force Office of Scientific Research (AFOSR) under agreement FA238616-1-4065.

${ }^{\dagger} 11$ Zafeiriou Rapesi St., Mesolonghi 30200, Greece (symeon.grivopoulos@gmail.com).

${ }^{\ddagger}$ Research School of Engineering, The Australian National University, Canberra ACT 2601, Australia (i.r.petersen@gmail.com).
} 
quantum information processing algorithms [23, 15, 32] and state generation [16, 19]. In the case that only the input/output relation of the LQSS is important, we have the problem of transfer function realization. This is the case, for example, in controller synthesis $[21,12,2]$.

In recent years, solutions have been proposed to both the strict and the transfer function realization problems. For the strict problem, [27, 25] propose a cascade of single-mode cavities realization. This allows for arbitrary couplings of the LQSS to its environment. However, not all possible interactions between cavity modes are possible, because the mode of a cavity can influence only modes of subsequent cavities. For this reason, direct Hamiltonian interactions [27] and feedback [25] between cavities have been used to "correct" the dynamics of the cascade to the desired form. For the transfer function realization problem, [30, 24] have shown that a cascade of singlemode cavities realization is possible for any passive LQSS, in which case all cavities needed to realize it are also passive. More recently in [26], it has been shown that a cascade of single-mode cavities realization is possible for generic LQSSs.

In this paper, we propose an alternative solution to the problem of transfer function realization. We show that by appropriate transformations of inputs and outputs (which can be realized experimentally by static linear optical networks; see subsection 2.3), one only needs to realize a much simpler transfer function. This "reduced" transfer function can be realized by a concatenation of single-mode cavities in a feedback interconnection through a static linear optical network. In the case of passive LQSSs, this realization is always possible, and all necessary devices needed for it are also passive.

In the case of passive LQSSs, the realization method employs crucially the singular value decomposition (SVD) for complex matrices. In order to extend the method to general LQSSs, we prove Theorem 4.1, which is an analogue of SVD for a class of even-dimensional structured matrices, the so-called doubled-up matrices [10, 31], in a class of complex spaces with indefinite scalar products, the so-called Krein spaces [6]. The role of the unitary matrices in the SVD, as isometries of the domain and target spaces of the linear map (matrix), is taken up by Bogoliubov matrices (see [10] and section 2) in the case of Krein spaces. This is an example of a new algebraic tool required by the theory of LQSSs (or linear quantum systems theory) that goes beyond the traditional toolbox of classic linear systems theory. The need for new tools and methods in quantum systems theory is to be expected, since quantum systems pose novel challenges compared to classical systems. Moreover, we expect that Theorem 4.1 in its equivalent form for symplectic spaces, namely, Theorem 4.8, will be of more general mathematical interest.

The rest of the paper is organized as follows: In section 2, we establish some notation and terminology used in the paper and provide a short overview of LQSSs and static linear optical devices and networks. In section 3, we demonstrate our method of transfer function realization for passive LQSSs, which is the simplest case. Finally, section 4 extends the realization result to general LQSSs using Theorem 4.1, which is the main technical result of this paper.

\section{Background material.}

\subsection{Notation and terminology.}

1. $x^{*}$ denotes the complex conjugate of a complex number $x$ or the adjoint of an operator $x$, respectively. As usual, re $x$ and $\operatorname{im} x$ denote the real and imaginary parts of a complex number. The commutator of two operators $X$ and $Y$ is defined as $[X, Y]=X Y-Y X$. 
2. For a matrix $X=\left[x_{i j}\right]$ with complex or operator entries, $X^{\#}=\left[x_{i j}^{*}\right], X^{\top}=$ $\left[x_{j i}\right]$ is the usual transpose, and $X^{\dagger}=\left(X^{\#}\right)^{\top}$. Also, for a vector $x=\left[x_{i}\right]$ with number or operator entries, we shall use the notation $\check{x}=\left(\begin{array}{c}x \\ x^{\#}\end{array}\right)$.

3. The identity matrix in $k$ dimensions will be denoted by $I_{k}$, and an $r \times s$ matrix of zeros will be denoted by $0_{r \times s} . \quad \delta_{i j}$ denotes the Kronecker delta symbol in $n$ dimensions, i.e., $I_{n}=\left[\delta_{i j}\right] . \operatorname{diag}\left(X_{1}, X_{2}, \ldots, X_{k}\right)$ is the blockdiagonal matrix formed by the square matrices $X_{1}, X_{2}, \ldots, X_{k}$. $\operatorname{Ker} X, \operatorname{Im} X$, and Rank $X$ denote, respectively, the kernel (null space), the image (range space), and the rank of a matrix $X$.

4. We define several matrices:

$$
\begin{aligned}
& J_{2 k}=\left(\begin{array}{cc}
I_{k} & 0_{k \times k} \\
0_{k \times k} & -I_{k}
\end{array}\right), \quad \Sigma_{2 k}=\left(\begin{array}{cc}
0_{k \times k} & I_{k} \\
I_{k} & 0_{k \times k}
\end{array}\right), \\
& \mathbb{J}_{2 k}=\left(\begin{array}{cc}
0_{k \times k} & I_{k} \\
-I_{k} & 0_{k \times k}
\end{array}\right), \quad \Phi_{2 k}=\frac{1}{\sqrt{2}}\left(\begin{array}{cc}
I_{k} & I_{k} \\
-\imath I_{k} & \imath I_{k}
\end{array}\right), \\
& \mathcal{S}_{k}=\left(\begin{array}{cccc}
0 & \cdots & 0 & 1 \\
0 & \cdots & 1 & 0 \\
\vdots & . & \vdots & \vdots \\
1 & \cdots & 0 & 0
\end{array}\right), \quad \sigma_{2}=\left(\begin{array}{cc}
0 & -\imath \\
\imath & 0
\end{array}\right) \text { (a Pauli matrix). }
\end{aligned}
$$

These matrices satisfy several useful identities:

$$
\begin{aligned}
& J_{2 k}^{2}=I_{2 k}, \quad \Sigma_{2 k}^{2}=I_{2 k}, \quad \Sigma_{2 k} J_{2 k} \Sigma_{2 k}=-J_{2 k}, \\
& \mathbb{J}_{2 k}^{2}=-I_{2 k}, \quad \mathcal{S}_{k}^{2}=I_{k}, \quad \mathbb{J}_{2 k} \mathcal{S}_{2 k} \mathbb{J}_{2 k}=\mathcal{S}_{2 k}, \\
& \Phi_{2 k}^{\dagger} \Phi_{2 k}=\Phi_{2 k} \Phi_{2 k}^{\dagger}=I_{2 k}, \quad \Phi_{2 k} J_{2 k} \Phi_{2 k}^{-1}=\imath \mathbb{J}_{2 k} .
\end{aligned}
$$

When their dimensions can be inferred from the context, we shall drop the various subscripts.

5. We shall denote by $j_{k}(\lambda)$ the upper Jordan block of size $k$ with eigenvalue $\lambda$ if $\lambda$ is real, and the direct sum of two Jordan blocks of size $k / 2$ each (for even $k$ ), the first with eigenvalue $\lambda$, and the second with eigenvalue $\lambda^{*}$, if $\lambda$ is complex. Then, $\mathbb{J}_{k} j_{k}(\lambda) \mathbb{J}_{k}=-j_{k}\left(\lambda^{*}\right)$ for even $k$. For a matrix $A$ with a real eigenvalue $\lambda$ of algebraic multiplicity $k$, or complex eigenvalues $\lambda$ and $\lambda^{*}$, of algebraic multiplicity $k / 2$ each (for even $k$ ), let $A V=V j_{k}(\lambda)$. $V$ will be called the eigenvector block corresponding to $\lambda$.

6. We define the Krein space $\left(\mathbb{C}^{2 k}, J_{2 k}\right)$ as the vector space $\mathbb{C}^{2 k}$ equipped with the indefinite inner product defined by $\langle v, w\rangle_{J}=v^{\dagger} J_{2 k} w$, for any $v, w \in \mathbb{C}^{2 k}$. The $J$-norm of a vector $v \in \mathbb{C}^{2 k}$ is defined by $|v|_{J}=\sqrt{\left|\langle v, v\rangle_{J}\right|}$, and if it is nonzero, a normalized multiple of $v$ is $v /|v|_{J}$. For a matrix $X \in \mathbb{C}^{2 r \times 2 s}$, considered as a map from $\left(\mathbb{C}^{2 s}, J_{2 s}\right)$ to $\left(\mathbb{C}^{2 r}, J_{2 r}\right)$, its adjoint operator will be called b-adjoint and denoted by $X^{b}$, to distinguish it from its usual adjoint $X^{\dagger}$. One can show that $X^{b}=J_{2 s} X^{\dagger} J_{2 r}$. The b-adjoint satisfies properties similar to those of the usual adjoint, namely $\left(x_{1} A+x_{2} B\right)^{b}=x_{1}^{*} A^{\mathrm{b}}+x_{2}^{*} B^{b}$, $(A B)^{b}=B^{b} A^{b}$, and $\left(A^{b}\right)^{b}=A$.

7. Given two matrices $X_{1}, X_{2} \in \mathbb{C}^{r \times s}$, we can form the matrix $X=\left(\begin{array}{ll}X_{1} & X_{2} \\ X_{2}^{\#} & X_{1}^{\#}\end{array}\right) \in$ $\mathbb{C}^{2 r \times 2 s}$. Such a matrix will be called doubled-up [10]. For $X \in \mathbb{C}^{2 r \times 2 s}$, $\Sigma_{2 r} X \Sigma_{2 s}=X^{\#}$ if and only if $X$ is doubled-up. When referring to a doubledup matrix $X, X_{1}$ and $X_{2}$ will denote its upper-left and upper-right blocks. Let 
us denote the set of doubled-up matrices in $\mathbb{C}^{2 r \times 2 s}$ by $\mathbb{C}_{d u}^{2 r \times 2 s} . \cup_{r, s=1}^{\infty} \mathbb{C}_{d u}^{2 r \times 2 s}$ is closed under addition, multiplication, and taking (b-)adjoints.

8. A matrix $R \in \mathbb{C}_{d u}^{2 k \times 2 k}$ is called Bogoliubov if it is b-unitary, i.e., $R R^{b}=R^{b} R=$ $I_{2 m}$. The set of these matrices forms a noncompact Lie group known as the Bogoliubov group. Bogoliubov matrices are isometries of Krein spaces.

2.2. Linear quantum stochastic systems. The material in this subsection is fairly standard, and our presentation aims mostly at establishing notation and terminology. To this end, we follow the papers [31, 34]. For the mathematical background necessary for a precise discussion of LQSSs, some standard references are $[29,22,13]$, while $[5,4]$ offer a physics perspective. The references $[27,3,9,8,10]$ contain a lot of relevant material as well.

The systems we consider in this paper are collections of quantum harmonic oscillators interacting among themselves, as well as with their environment. The $i$ th harmonic oscillator $(i=1, \ldots, n)$ is described by its position and momentum variables, $x_{i}$ and $p_{i}$, respectively. These are self-adjoint operators satisfying the canonical commutation relations (CCRs) $\left[x_{i}, x_{j}\right]=0,\left[p_{i}, p_{j}\right]=0$, and $\left[x_{i}, p_{j}\right]=\imath \delta_{i j}$ for $i, j=1, \ldots, n$. We find it more convenient to work with the so-called annihilation and creation operators $a_{i}=\frac{1}{\sqrt{2}}\left(x_{i}+\imath p_{i}\right)$ and $a_{i}^{*}=\frac{1}{\sqrt{2}}\left(x_{i}-\imath p_{i}\right)$. They satisfy the CCRs $\left[a_{i}, a_{j}\right]=0,\left[a_{i}^{*}, a_{j}^{*}\right]=0$, and $\left[a_{i}, a_{j}^{*}\right]=\delta_{i j}, i, j=1, \ldots, n$. In what follows, $a=\left(a_{1}, a_{2}, \ldots, a_{n}\right)^{\top}$.

The environment is modelled as a collection of zero temperature bosonic quantum fields. The $i$ th quantum field $(i=1, \ldots, m)$ is described by the bosonic field annihilation and creation operators $\mathcal{A}_{i}(t)$ and $\mathcal{A}_{i}^{*}(t)$, respectively. The field operators are adapted quantum stochastic processes with forward differentials $d \mathcal{A}_{i}(t)=\mathcal{A}_{i}(t+$ $d t)-\mathcal{A}_{i}(t)$ and $d \mathcal{A}_{i}^{*}(t)=\mathcal{A}_{i}^{*}(t+d t)-\mathcal{A}_{i}^{*}(t)$. They satisfy the quantum Itô products $d \mathcal{A}_{i}(t) d \mathcal{A}_{j}(t)=0, d \mathcal{A}_{i}^{*}(t) d \mathcal{A}_{j}^{*}(t)=0, d \mathcal{A}_{i}^{*}(t) d \mathcal{A}_{j}(t)=0$, and $d \mathcal{A}_{i}(t) d \mathcal{A}_{j}^{*}(t)=\delta_{i j} d t$. In what follows, $\mathcal{A}=\left(\mathcal{A}_{1}, \mathcal{A}_{2}, \ldots, \mathcal{A}_{m}\right)^{\top}$.

To describe the dynamics of the harmonic oscillators and the quantum fields (noises), we need to introduce certain operators. We begin with the class of annihilator only LQSSs. We also refer to such systems as passive LQSSs, because systems in this class describe optical devices, such as damped optical cavities, that do not require an external source of quanta for their operation. First, we have the Hamiltonian operator $H=a^{\dagger} \Omega a$, which specifies the dynamics of the harmonic oscillators in the absence of any environmental influence. $\Omega \in \mathbb{C}^{n \times n}$ is a Hermitian matrix referred to as the Hamiltonian matrix. Next, we have the coupling operator $L$ (vector of operators) that specifies the interaction of the harmonic oscillators with the quantum fields. $L$ depends linearly on the annihilation operators and can be expressed as $L=C a . C \in \mathbb{C}^{m \times n}$ is called the coupling matrix. Finally, we have the unitary scattering matrix $S \in \mathbb{C}^{m \times m}$, which describes the interactions between the quantum fields themselves. In practice, it represents the unitary transformation effected on the field modes by a static passive linear optical network that precedes the LQSS; see subsection 2.3.

In the Heisenberg picture of quantum mechanics, the joint evolution of the harmonic oscillators and the quantum fields is described by the following system of quantum stochastic differential equations (QSDEs):

$$
\begin{aligned}
d a & =\left(-\imath \Omega-\frac{1}{2} C^{\dagger} C\right) a d t-C^{\dagger} S d \mathcal{A}, \\
d \mathcal{A}_{\text {out }} & =C a d t+S d \mathcal{A} .
\end{aligned}
$$

The field operators $\mathcal{A}_{\text {iout }}(t), i=1, \ldots, m$, describe the outputs of the system. We 
can generalize (1a) and (1b) by allowing the system inputs not to be just quantum noises, but to contain a "signal part," as well. Such is the case when the output of a passive LQSS is fed into another passive LQSS. So we substitute the more general notation $\mathcal{U}$ and $\mathcal{Y}$ for $\mathcal{A}$ and $\mathcal{A}_{\text {out }}$, respectively. The forward differentials $d \mathcal{U}$ and $d \mathcal{Y}$ of $m$-dimensional inputs and outputs, respectively, contain quantum noises, as well as linear combinations of variables of other systems. The resulting QSDEs are the following:

$$
\begin{aligned}
& d a=\left(-\imath \Omega-\frac{1}{2} C^{\dagger} C\right) a d t-C^{\dagger} S d \mathcal{U}, \\
& d \mathcal{Y}=C a d t+S d \mathcal{U} .
\end{aligned}
$$

One can show that the structure of $(2 \mathrm{a})$ and $(2 \mathrm{~b})$ is preserved under linear transformations of the state $\hat{a}=V a$ if and only if $V$ is unitary. Under such a state transformation, the system parameters $(S, C, \Omega)$ transform according to $(\hat{S}, \hat{C}, \hat{\Omega})=\left(S, C V^{-1}, V \Omega V^{\dagger}\right)$. From the point of view of quantum mechanics, $V$ must be unitary so that the new annihilation and creation operators satisfy the correct CCRs.

General LQSSs may contain active devices that require an external source of quanta for their operation, such as degenerate parametric amplifiers. In this case, system and field creation operators appear in the QSDEs for system and field annihilation operators, and vice versa. Since these are adjoint operators which have to be treated as separate variables, this leads to the appearance of doubled-up matrices in the corresponding QSDEs. To describe the most general linear dynamics of harmonic oscillators and quantum noises, we introduce generalized versions of the Hamiltonian operator, the coupling operator, and the scattering matrix defined above. We begin with the Hamiltonian operator

$$
H=\frac{1}{2}\left(\begin{array}{l}
a \\
a^{\#}
\end{array}\right)^{\dagger}\left(\begin{array}{cc}
\Omega_{1} & \Omega_{2} \\
\Omega_{2}^{\#} & \Omega_{1}^{\#}
\end{array}\right)\left(\begin{array}{l}
a \\
a^{\#}
\end{array}\right)=\frac{1}{2} \check{a}^{\dagger} \Omega \check{a},
$$

which specifies the dynamics of the harmonic oscillators in the absence of any environmental influence. The Hamiltonian matrix $\Omega \in \mathbb{C}_{d u}^{2 n \times 2 n}$ is Hermitian. Next, we have the coupling operator $L$, which specifies the interaction of the harmonic oscillators with the quantum fields. $L$ depends linearly on the creation and annihilation operators, $L=C_{1} a+C_{2} a^{\#}$. We construct the coupling matrix $C \in \mathbb{C}_{d u}^{2 m \times 2 n}$ from $C_{1} \in \mathbb{C}^{m \times n}$ and $C_{2} \in \mathbb{C}^{m \times n}$. Finally, we have the Bogoliubov generalized scattering matrix $S \in \mathbb{C}_{d u}^{2 m \times 2 m}$, which describes the interactions between the quantum fields themselves. In practice, it represents the Bogoliubov transformation effected on the field modes by a general static linear quantum optical network that precedes the LQSS; see subsection 2.3 and [10].

In the Heisenberg picture of quantum mechanics, the joint evolution of the harmonic oscillators and the quantum fields is described by the following system of quantum stochastic differential equations (QSDEs):

$$
\begin{aligned}
d \check{a} & =\left(-\imath J \Omega-\frac{1}{2} C^{b} C\right) \check{a} d t-C^{b} S d \check{\mathcal{U}}, \\
d \check{\mathcal{Y}} & =C \check{a} d t+S d \check{\mathcal{U}} .
\end{aligned}
$$

The forward differentials $d \mathcal{U}$ and $d \mathcal{Y}$ of $m$-dimensional inputs and outputs, respectively, contain quantum noises, as well as a signal part (linear combinations of vari- 
ables of other systems). One can show that the structure of (3a) and (3b) is preserved under linear transformations of the state $\check{\hat{a}}=V \check{a}$ if and only if $V$ is Bogoliubov. In that case the system parameters $(S, C, \Omega)$ transform according to $(\hat{S}, \hat{C}, \hat{\Omega})=$ $\left(S, C V^{-1},\left(V^{-1}\right)^{\dagger} \Omega V^{-1}\right)$. From the point of view of quantum mechanics, $V$ must be Bogoliubov so that the new annihilation and creation operators satisfy the correct CCRs.

We end this subsection with the model of the single-mode optical cavity, which is the basic device for the proposed realization method in this paper. It is described by its optical mode $a$, with Hamiltonian matrix $\Omega=\operatorname{diag}(\Delta, \Delta)$, where $\Delta \in \mathbb{R}$ is the so-called cavity detuning. For a cavity with $m$ inputs and outputs, we let $C_{1}=\left(e^{\imath \varphi_{1}} \sqrt{\kappa_{1}}, \ldots, e^{\imath \varphi_{m}} \sqrt{\kappa_{m}}\right)^{\top}$ and $C_{2}=\left(e^{i \theta_{1}} \sqrt{g_{1}}, \ldots, e^{\imath \theta_{m}} \sqrt{g_{m}}\right)^{\top} \cdot \kappa_{i}$ and $g_{i}$ will be called the passive and the active coupling coefficients of the $i$ th quantum noise to the cavity, respectively. When $g_{i}=0$, the interaction of the cavity mode with the $i$ th quantum noise (along with the port) will be referred to as (purely) passive, and when $\kappa_{i}=0$, it will be referred to as (purely) active. The model of a cavity with $m$ inputs and outputs is the following:

$$
\begin{aligned}
d a & =\left[-\imath \Delta-\frac{1}{2}\left(C_{1}^{\dagger} C_{1}-C_{2}^{\top} C_{2}^{\#}\right)\right] a d t-C_{1}^{\dagger} d \mathcal{U}+C_{2}^{\top} d \mathcal{U}^{\#} \\
& =\left(-\imath \Delta-\frac{\gamma}{2}\right) a d t+\sum_{i=1}^{m}\left(-e^{-\imath \varphi_{i}} \sqrt{\kappa_{i}} d \mathcal{U}_{i}+e^{\imath \theta_{i}} \sqrt{g_{i}} d \mathcal{U}_{i}^{\#}\right), \\
d \mathcal{Y}_{i} & =e^{\imath \varphi_{i}} \sqrt{\kappa_{i}} a d t+e^{\imath \theta_{i}} \sqrt{g_{i}} a^{\#} d t+d \mathcal{U}_{i},
\end{aligned}
$$

$i=1, \ldots, m$, where $\gamma=\sum_{i=1}^{m}\left(\kappa_{i}-g_{i}\right)$. If a quantum noise couples passively to the cavity, the corresponding interaction may be realized with a partially transmitting mirror. For an interaction that has an active component, a more complicated implementation is needed; see, e.g., [27, subsection 6.3] for the details. From now on, we shall use the system-theoretic term port for any part of the experimental setup that realizes an interaction of the cavity mode with a quantum noise (where an input enters and an output exits the cavity).

2.3. Static linear optical devices and networks. In addition to the generalized cavities discussed above, our proposed realization method for LQSSs makes use of static linear quantum optical devices and networks, as well. Useful references for this material are $[17,27,18,1]$. The most basic such devices are the following:

1. The phase shifter: This device produces a phase shift in its input optical field. That is, if $\mathcal{U}$ and $\mathcal{Y}$ are its input and output fields, respectively, then $\mathcal{Y}=e^{\imath \theta} \mathcal{U}$. Notice that $\mathcal{Y}^{*} \mathcal{Y}=\mathcal{U}^{*} \mathcal{U}$. This means that the energy of the output field is equal to that of the input field, and hence energy is conserved. Such a device is called passive.

2. The beam splitter: This device produces linear combinations of its two input fields. If we denote its inputs by $\mathcal{U}_{1}$ and $\mathcal{U}_{2}$, and its outputs by $\mathcal{Y}_{1}$ and $\mathcal{Y}_{2}$, then

$$
\left(\begin{array}{l}
\mathcal{Y}_{1} \\
\mathcal{Y}_{2}
\end{array}\right)=R\left(\begin{array}{l}
\mathcal{U}_{1} \\
\mathcal{U}_{2}
\end{array}\right)
$$

where

$$
R=\left(\begin{array}{ccc}
e^{\imath \frac{\varphi+\psi}{2}} \cos \frac{\theta}{2} & e^{\imath \frac{\psi-\varphi}{2}} \sin \frac{\theta}{2} \\
-e^{\imath \frac{\varphi-\psi}{2}} \sin \frac{\theta}{2} & e^{-\imath \frac{\varphi+\psi}{2}} \cos \frac{\theta}{2}
\end{array}\right) .
$$

Copyright $@$ ㅇ by SIAM. Unauthorized reproduction of this article is prohibited. 
$0 \leq \theta<2 \pi$ is called the mixing angle of the beam splitter. $\varphi$ and $\psi$ are phase differences in the two input and the two output fields, respectively, produced by phase shifters. This form of $R$ corresponds to a general $2 \times 2$ unitary matrix of determinant +1 . We can see that

$$
\left(\begin{array}{ll}
\mathcal{Y}_{1}^{*} & \mathcal{Y}_{2}^{*}
\end{array}\right)\left(\begin{array}{l}
\mathcal{Y}_{1} \\
\mathcal{Y}_{2}
\end{array}\right)=\left(\begin{array}{ll}
\mathcal{U}_{1}^{*} & \mathcal{U}_{2}^{*}
\end{array}\right) R^{\dagger} R\left(\begin{array}{l}
\mathcal{U}_{1} \\
\mathcal{U}_{2}
\end{array}\right)=\left(\begin{array}{ll}
\mathcal{U}_{1}^{*} & \mathcal{U}_{2}^{*}
\end{array}\right)\left(\begin{array}{l}
\mathcal{U}_{1} \\
\mathcal{U}_{2}
\end{array}\right)
$$

and hence the total energy of the output fields is equal to that of the input fields. So, the beam splitter is also a passive device.

3. The squeezer: This reduces the variance in the real quadrature $\left(\mathcal{U}+\mathcal{U}^{*}\right) / 2$, or the imaginary quadrature $\left(\mathcal{U}-\mathcal{U}^{*}\right) / 2 \imath$ of an input field $\mathcal{U}$, while increasing the variance in the other quadrature. Its operation is described by

$$
\left(\begin{array}{c}
\mathcal{Y} \\
\mathcal{Y}^{*}
\end{array}\right)=R\left(\begin{array}{c}
\mathcal{U} \\
\mathcal{U}^{*}
\end{array}\right)
$$

where

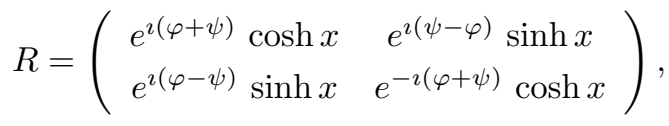

$x \in \mathbb{R}$ is the squeezing parameter, and $\varphi, \psi$ are phase shifts in the input and the output fields, respectively, produced by phase shifters. This form of $R$ corresponds to a general $2 \times 2$ Bogoliubov matrix. As $\mathcal{Y}^{*} \mathcal{Y} \neq \mathcal{U}^{*} \mathcal{U}$ for $x \neq 0$, the squeezer is an active device.

By connecting various static linear optical devices, we may form static linear optical networks (multiport devices). For a passive network, i.e., one composed solely of passive devices, we have that $\mathcal{Y}=R \mathcal{U}$, where $R$ is an $m \times m$ unitary matrix. Such a network is a generalization of the beam splitter and is sometimes called a multibeam splitter. It turns out that any passive static network can be constructed exclusively from phase shifters and beam splitters [33]. In the general case, where the network may contain active devices as well, we have the more general relation $\check{\mathcal{Y}}=R \check{\mathcal{U}}$, where $R$ is a $2 m \times 2 m$ Bogoliubov matrix. Using the Bloch-Messiah reduction (factorization) of a Bogoliubov matrix [27, 18, 1], it turns out that a general static network can be constructed exclusively from phase shifters, beam splitters, and squeezers.

Figure 1 contains graphical representations of a multiport cavity and two static networks that will be used in what follows.

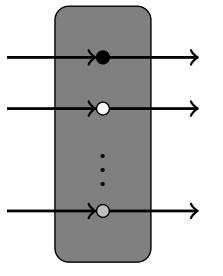

(a)

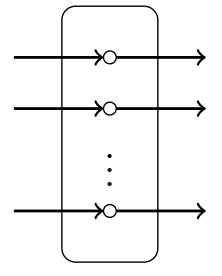

(b)

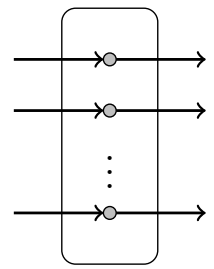

(c)

FIG. 1. (a) The gray block represents a multiport cavity. Black, white, and gray discs represent active, passive, and general ports, respectively. (b)-(c) A passive and a general static network, respectively. 


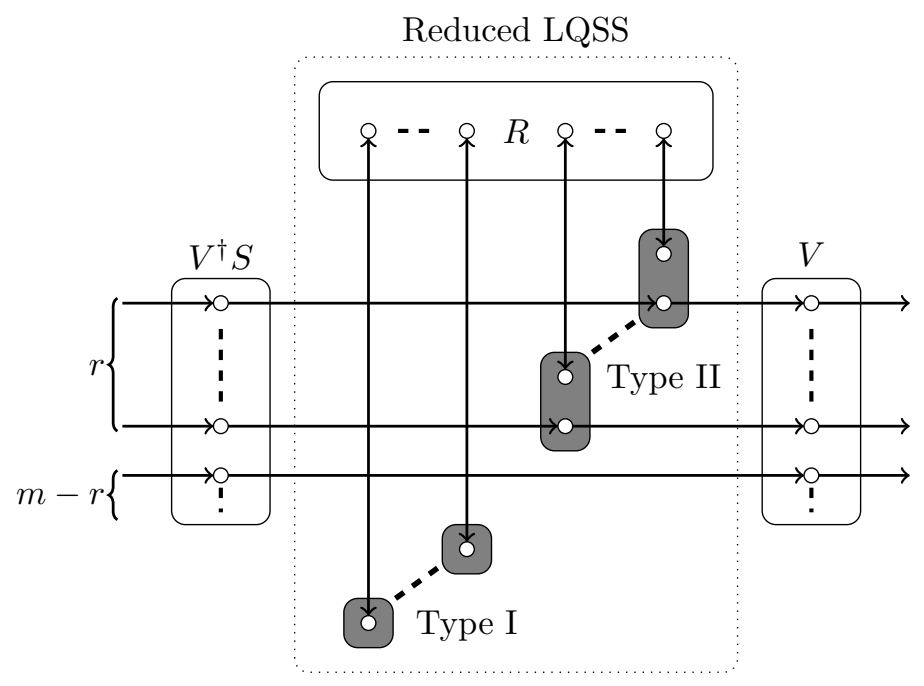

FIG. 2. Graphical representation of a particular LQSS architecture, used to realize the transfer functions of arbitrary passive LQSSs.

3. Transfer function realization of passive LQSSs. We present our realization method of transfer functions of LQSSs in the case of passive systems first, because it is the simplest one. Let us refer to Figure 2. It represents a particular configuration, composed of a pre- and a postprocessing passive static linear network, and a concatenation of single-mode optical cavities in a feedback connection through a third passive static linear network. There are two types of cavities: Type I cavities have a single passive port that connects to the static network, while Type II also have a second passive port whose input and output connect to an output and an input, respectively, of the pre- and the postprocessing network. Ports used for feedback will be referred to as interconnection ports, while ports connected to inputs and outputs of the pre- and the postprocessing networks will be referred to as system ports. The part of the configuration between the pre- and the postprocessing networks will be referred to as the "reduced LQSS." In what follows, we prove that this sort of configuration can realize the transfer function of an arbitrary passive LQSS for an appropriate choice of parameters.

TheOREM 3.1. Given a passive LQSS with parameters $(S, C, \Omega)$, let

$$
G(s)=\left[I-C\left(s I+\imath \Omega+\frac{1}{2} C^{\dagger} C\right)^{-1} C^{\dagger}\right] S
$$

be its transfer function. Let $C=V \hat{C} W^{\dagger}$ be the $S V D$ of the coupling matrix $C$, with

$$
\hat{C}=\left(\begin{array}{ccc|c}
\sqrt{\kappa}_{1} & & & \\
& \ddots & & 0 \\
& & \sqrt{\kappa}_{r} & \\
\hline 0 & & 0
\end{array}\right),
$$

where $r \leq \min \{n, m\}$. Then, $G(s)$ can be factorized as $G(s)=V \hat{G}(s)\left(V^{\dagger} S\right)$, where 
$\hat{G}(s)$ has the form

$$
\hat{G}(s)=I-\hat{C}\left(s I+\imath \hat{\Omega}+\frac{1}{2} \hat{C}^{\dagger} \hat{C}\right)^{-1} \hat{C}^{\dagger},
$$

with $\hat{\Omega}=W^{\dagger} \Omega W$. $\hat{G}(s)$ is the transfer function of a passive LQSS with parameters $(I, \hat{C}, \hat{\Omega})$. If we let $D=\operatorname{diag}\left(\Delta_{1}, \ldots, \Delta_{n}\right)$ and $\tilde{C}=\operatorname{diag}\left(\sqrt{\tilde{\kappa}_{1}}, \ldots, \sqrt{\tilde{\kappa}_{n}}\right)$, where $\Delta_{i} \in$ $\mathbb{R}$ and $\tilde{\kappa}_{i}>0$, then $\hat{G}(s)$ can be realized by the following feedback network of $n-r$ one-port and $r$ two-port cavities:

$$
\begin{aligned}
d a & =\left(-\imath D-\frac{1}{2} \tilde{C}^{\dagger} \tilde{C}-\frac{1}{2} \hat{C}^{\dagger} \hat{C}\right) a d t-\tilde{C}^{\dagger} d \mathcal{U}_{i n t}-\hat{C}^{\dagger} d \mathcal{U}, \\
d \mathcal{Y} & =\hat{C} a d t+d \mathcal{U} \\
d \mathcal{Y}_{i n t} & =\tilde{C} a d t+d \mathcal{U}_{i n t}, \\
d \mathcal{U}_{i n t} & =R d \mathcal{Y}_{i n t} .
\end{aligned}
$$

The unitary matrix $R$ (feedback gain) is determined through the relations

$$
\begin{aligned}
X & =2 \imath \tilde{C}^{-\dagger}(\hat{\Omega}-D) \tilde{C}^{-1}, \\
R & =(X-I)(X+I)^{-1} .
\end{aligned}
$$

The $m$-dimensional vectors $\mathcal{U}$ and $\mathcal{Y}$ contain the inputs and outputs of the system ports, and the $n$-dimensional vectors $\mathcal{U}_{\text {int }}$ and $\mathcal{Y}_{\text {int }}$ contain the inputs and outputs of the interconnection ports. $D_{i}$ and $\tilde{\kappa}_{i}$ are the cavity detuning and the coupling coefficient of the interconnection port, respectively, of the $i$ th cavity, which can be chosen arbitrarily. From the fact that $D, \hat{C}$, and $\tilde{C}$ are diagonal, all diagonal elements of $\tilde{C}$ are nonzero, and only $r$ diagonal elements of $\hat{C}$ are nonzero, we see that (5a)-(5d) describe a collection of cavities, all of which have one interconnection port, but only $r$ have system ports. Hence, the feedback network consists of $n-r$ one-port and $r$ two-port cavities.

Proof of Theorem 3.1. It suffices to prove that $\hat{G}(s)$ is the transfer function of the system described by $(5 \mathrm{a})-(5 \mathrm{~d})$. To this end, we combine the last two equations in $(5 \mathrm{~d})$ to obtain the relation

$$
d \mathcal{U}_{\text {int }}=(I-R)^{-1} R \tilde{C} a d t .
$$

Now, we introduce the Cayley transform [7]

$$
X=(I-R)^{-1}(I+R),
$$

defined for matrices $R$ without unit eigenvalues. The unitarity of $R$ implies that $X$ is skew-Hermitian. We can also solve uniquely for $R$ in terms of $X$ with the following result:

$$
R=(X-I)(X+I)^{-1} .
$$

$R$ can be seen to be unitary due to the skew-Hermitian nature of $X$. Also, it is easy to see that $(I-R)^{-1} R=-\frac{1}{2} I+\frac{1}{2} X$. Using the relation between $d \mathcal{U}_{\text {int }}$ and $a$, and the definition of $X$, the equations for the reduced LQSS take the following form:

$$
\begin{aligned}
d a & =\left(-\imath D-\frac{1}{2} \tilde{C}^{\dagger} X \tilde{C}-\frac{1}{2} \hat{C}^{\dagger} \hat{C}\right) a d t-\hat{C}^{\dagger} d \mathcal{U}, \\
d \mathcal{Y} & =\hat{C} a d t+d \mathcal{U}
\end{aligned}
$$

Copyright $@$ by SIAM. Unauthorized reproduction of this article is prohibited. 
These equations describe a passive LQSS with Hamiltonian matrix $\hat{\Omega}$ given by the expression

$$
\hat{\Omega}=D-\frac{\imath}{2} \tilde{C}^{\dagger} X \tilde{C}
$$

Given any values for the cavity parameters $\Delta_{i} \in \mathbb{R}$ and $\tilde{\kappa}_{i}>0$, and any desired Hamiltonian matrix $\hat{\Omega}=W^{\dagger} \Omega W$, we may determine the unique $X$ (and hence the unique $R$ ) that produces this $\hat{\Omega}$ by the expression

$$
X=2 \imath \tilde{C}^{-\dagger}(\hat{\Omega}-D) \tilde{C}^{-1} .
$$

This completes the proof.

Remark 3.2. If the unitary feedback gain $R$ is block-diagonal, i.e., if $R=$ $\operatorname{diag}\left(R_{r}, R_{n-r}\right)$, where $R_{r}$ and $R_{n-r}$ are unitary $r \times r$ and $(n-r) \times(n-r)$ matrices, respectively, then the $n-r$ modes that are not influenced directly by the inputs are uncontrollable and unobservable. Indeed, it can be proved that this is a necessary and sufficient condition for a passive LQSS to lose controllability and observability. (The two system properties are equivalent for passive LQSSs; see [40].)

3.1. Illustrative example. Consider the three-mode, three-port passive LQSS with the following parameters:

$$
\Omega=\left(\begin{array}{rrr}
5 & 1 & -2 \\
1 & 3 & 0 \\
-2 & 0 & 4
\end{array}\right), \quad C=\left(\begin{array}{rrr}
1 & 2 & 1 \\
0 & -1 & 3 \\
2 & 3 & 5
\end{array}\right), \quad \text { and } S=I_{3} .
$$

The SVD of $C$ is given by $C=V \hat{C} W^{\dagger}$, with

$$
V=\left(\begin{array}{rrr}
-0.2987 & 0.4941 & -0.8165 \\
-0.3065 & -0.8599 & -0.4082 \\
-0.9038 & 0.1283 & 0.4082
\end{array}\right), \quad W=\left(\begin{array}{rrr}
-0.3093 & 0.2717 & -0.9113 \\
-0.4409 & 0.8081 & 0.3906 \\
-0.8426 & -0.5226 & 0.1302
\end{array}\right) \text {, }
$$

and $\hat{C}=\operatorname{diag}(6.8092,2.7632,0)$. The Hamiltonian of the reduced LQSS is given by

$$
\hat{\Omega}=W^{\dagger} \Omega W=\left(\begin{array}{rrr}
3.1315 & 0.0370 & -0.7200 \\
0.0370 & 4.4278 & -2.2169 \\
-0.7200 & -2.2169 & 4.4407
\end{array}\right) .
$$

Letting $D=0_{3 \times 3}$ and $\tilde{C}=I_{3},(6)$ produces the following $X$ :

$$
X=\imath\left(\begin{array}{rrr}
6.2631 & 0.0740 & -1.4400 \\
0.0740 & 8.8556 & -4.4337 \\
-1.4400 & -4.4337 & 8.8814
\end{array}\right) \text {, }
$$

from which we calculate the feedback gain $R$ using (7), to be equal to

$$
R=\left(\begin{array}{rrr}
0.9429+0.3245 \imath & -0.0145+0.0276 \imath & -0.0237+0.0637 \imath \\
-0.0145+0.0276 \imath & 0.9438+0.2918 \imath & -0.0467+0.1449 \imath \\
-0.0237+0.0637 \imath & -0.0467+0.1449 \imath & 0.9389+0.3010 \imath
\end{array}\right) .
$$

4. Transfer function realization of general LQSSs. In this section, we present our synthesis method for the case of general LQSSs. To proceed as in section 3, we first derive an analogue of the SVD for doubled-up matrices in Krein spaces.

Copyright $@$ by SIAM. Unauthorized reproduction of this article is prohibited. 


\subsection{A canonical form for doubled-up matrices in Krein spaces.}

TheOrem 4.1. Let $C=\left(\begin{array}{cc}C_{1} & C_{2} \\ C_{2}^{\#} & C_{1}^{\#}\end{array}\right) \in \mathbb{C}_{d u}^{2 m \times 2 n}$ and $\mathcal{C} \doteq C^{b} C$. We assume that all the eigenvalues of $\mathcal{C}$ are semisimple, and that $\operatorname{Ker} \mathcal{C}=\operatorname{Ker} C$. Let $\lambda_{i}^{+}>0, i=$ $1, \ldots, r_{+}, \lambda_{i}^{-}<0, i=1, \ldots, r_{-}$, and $\lambda_{i}^{c}, i=1 \ldots, r_{c}$, with $\operatorname{im} \lambda_{i}^{c}>0$, be the nontrivial eigenvalues of $\mathcal{C}$ (along with $\lambda_{i}^{c *}$ ). Then, there exist Bogoliubov matrices $V \in \mathbb{C}_{d u}^{2 m \times 2 m}$ and $W \in \mathbb{C}_{d u}^{2 n \times 2 n}$ and a matrix $\hat{C}=\left(\begin{array}{c}\hat{C}_{1} \hat{C}_{2} \\ \hat{C}_{2}^{\#} \hat{C}_{1}^{\#}\end{array}\right) \in \mathbb{C}_{d u}^{2 m \times 2 n}$, such that $C=V \hat{C} W^{b}$, with $\hat{C}_{1}=\left(\begin{array}{cc}\bar{C}_{1} & 0 \\ 0 & 0\end{array}\right), \hat{C}_{2}=\left(\begin{array}{cc}\bar{C}_{2} & 0 \\ 0 & 0\end{array}\right) \in \mathbb{C}^{r \times r}\left(r=r_{+}+r_{-}+2 r_{c} \leq \min \{n, m\}\right)$, and

$$
\begin{aligned}
& \bar{C}_{1}=\operatorname{diag}(\sqrt{\lambda_{1}^{+}}, \ldots, \sqrt{\lambda_{r_{+}}^{+}}, \underbrace{0, \ldots, 0}_{r_{-}}, \alpha_{1} I_{2}, \ldots, \alpha_{r_{c}} I_{2}), \\
& \bar{C}_{2}=\operatorname{diag}(\underbrace{0, \ldots, 0}_{r_{+}}, \sqrt{\left|\lambda_{1}^{-}\right|}, \ldots, \sqrt{\left|\lambda_{r_{-}}^{-}\right|},-\beta_{1} \sigma_{2}, \ldots,-\beta_{r_{c}} \sigma_{2}) .
\end{aligned}
$$

$\alpha_{i}$ and $\beta_{i}$ are calculated in terms of $\lambda_{i}^{c}, i=1 \ldots, r_{c}$, by

$$
\alpha_{i}=\sqrt{\frac{\left|\lambda_{i}^{c}\right|+\operatorname{re} \lambda_{i}^{c}}{2}} \quad \text { and } \quad \beta_{i}=\frac{\operatorname{im} \lambda_{i}^{c}}{\sqrt{2\left(\left|\lambda_{i}^{c}\right|+\operatorname{re} \lambda_{i}^{c}\right)}} .
$$

The matrix $\mathcal{C}=C^{b} C$ in the theorem is b-Hermitian $\left(\mathcal{C}^{b}=\mathcal{C}\right)$ and doubledup $\left(\Sigma \mathcal{C} \Sigma=\mathcal{C}^{\#}\right)$. The following two lemmas establish spectral properties of such matrices, necessary for the proof of Theorem 4.1.

Lemma 4.2. Let $A$ be a b-Hermitian matrix in $\left(\mathbb{C}^{2 n}, J\right)$, let $\lambda_{1}, \ldots, \lambda_{A}$ be its real eigenvalues, and let $\lambda_{A+1}, \ldots, \lambda_{B}$ be its complex eigenvalues with positive imaginary parts. There exists an invertible matrix $Z \in \mathbb{C}^{2 n \times 2 n}$, such that

$$
\begin{aligned}
Z^{-1} A Z & =\hat{A}, \\
Z^{\dagger} J Z & =\hat{J},
\end{aligned}
$$

where

$$
\begin{aligned}
& \hat{A} \doteq \operatorname{diag}\left(j_{k_{1}}\left(\lambda_{1}\right), \ldots, j_{k_{A}}\left(\lambda_{A}\right), j_{k_{A+1}}\left(\lambda_{A+1}\right), \ldots, j_{k_{B}}\left(\lambda_{B}\right)\right), \\
& \hat{J} \doteq \operatorname{diag}\left(\varepsilon_{1} \mathcal{S}_{k_{1}}, \ldots, \varepsilon_{A} \mathcal{S}_{k_{A}}, \varepsilon_{A+1} \mathcal{S}_{k_{A+1}}, \ldots, \varepsilon_{B} \mathcal{S}_{k_{B}}\right),
\end{aligned}
$$

with $\varepsilon_{i}= \pm 1, i=1, \ldots, A$, and $\varepsilon_{i}=1, i=A+1, \ldots, B$. This decomposition is unique, except for eigenvalue permutations and multiplications of eigenvector blocks (submatrices of $Z$ ) by phase factors.

Proof. The lemma is just the spectral theorem for self-adjoint matrices in spaces with indefinite scalar products [6], applied to the case of a b-Hermitian matrix in the Krein space $\left(\mathbb{C}^{2 n}, J\right)$.

Lemma 4.3. Let $A$ be a b-Hermitian, doubled-up matrix in $\left(\mathbb{C}^{2 n}, J\right)$, and let $V$ be an eigenvector block corresponding to the eigenvalue $\lambda$. Then, $\Sigma V^{\#}$ if $\lambda \in \mathbb{R}$, or $\Sigma V^{\#} \mathbb{J}_{k}$ if $\lambda \in \mathbb{C}-\mathbb{R}$, is a different eigenvector block corresponding to $\lambda$.

Proof. Let $V$ be an eigenvector block corresponding to the eigenvalue $\lambda$. Then, from (10a) and (10b), we have

$$
\begin{aligned}
A V & =V j_{k}(\lambda), \\
V^{\dagger} J V & =\varepsilon_{k} \mathcal{S}_{k} .
\end{aligned}
$$

Copyright $\odot$ by SIAM. Unauthorized reproduction of this article is prohibited. 
Using the properties of $\Sigma$ and doubled-up matrices, we compute

$$
\begin{gathered}
(\Sigma A \Sigma)(\Sigma V)=(\Sigma V) j_{k}(\lambda) \Rightarrow A^{\#}(\Sigma V)=(\Sigma V) j_{k}(\lambda) \\
\Rightarrow A\left(\Sigma V^{\#}\right)=\left(\Sigma V^{\#}\right) j_{k}\left(\lambda^{*}\right), \\
(\Sigma V)^{\dagger}(\Sigma J \Sigma)(\Sigma V)=\varepsilon_{k} \mathcal{S}_{k} \Rightarrow(\Sigma V)^{\dagger}(-J)(\Sigma V)=\varepsilon_{k} \mathcal{S}_{k} \\
\Rightarrow\left(\Sigma V^{\#}\right)^{\dagger} J\left(\Sigma V^{\#}\right)=-\varepsilon_{k} \mathcal{S}_{k} .
\end{gathered}
$$

When $\lambda$ is real, (12a) implies that $\Sigma V^{\#}$ is also an eigenvector block of $\lambda$, and (12b) implies that $V$ and $\Sigma V^{\#}$ cannot be related by a simple phase factor; hence they are different. When $\lambda$ is complex (hence, $k$ is even), (12a) and (12b) can be rewritten as follows:

$$
\begin{gathered}
A\left(\Sigma V^{\#}\right)=-\left(\Sigma V^{\#}\right) \mathbb{J}_{k} j_{k}(\lambda) \mathbb{J}_{k} \Rightarrow A\left(\Sigma V^{\#} \mathbb{J}_{k}\right)=\left(\Sigma V^{\#} \mathbb{J}_{k}\right) j_{k}(\lambda), \\
\left(\Sigma V^{\#}\right)^{\dagger} J\left(\Sigma V^{\#}\right)=-\mathbb{J}_{k} \mathcal{S}_{k} \mathbb{J}_{k} \Rightarrow\left(\Sigma V^{\#} \mathbb{J}_{k}\right)^{\dagger} J\left(\Sigma V^{\#} \mathbb{J}_{k}\right)=\mathcal{S}_{k} .
\end{gathered}
$$

Hence, $\Sigma V^{\#} \mathbb{J}_{k}$ is an eigenvector block corresponding to $\lambda$. To show that it is different from $V$, let $\Sigma V^{\#} \mathbb{J}_{k}=\rho V$, where $\rho$ is a phase factor (hence, $\rho^{*}=1 / \rho=-\rho$ ). From this, it is easy to show that $V=-|\rho|^{2} V$, which implies $V=0$, a contradiction. Hence, $\Sigma V^{\#} \mathbb{J}_{k}$ is different from $V$.

Proof of Theorem 4.1. We begin with the positive eigenvalues, $\lambda_{i}^{+}, i=1, \ldots, r_{+}$. From Lemma 4.3, there correspond two eigenvectors to each eigenvalue: $\zeta_{i}$, with $\zeta_{i}^{\dagger} J \zeta_{i}=1$, and $\Sigma \zeta_{i}^{\#}$, with $\left(\Sigma \zeta_{i}^{\#}\right)^{\dagger} J\left(\Sigma \zeta_{i}^{\#}\right)=-1$. (We adopt the convention of expressing the eigenvector whose inner product with itself is negative in terms of the eigenvector whose inner product with itself is positive.) These two eigenvectors are also $J$-orthogonal to each other, i.e., $\zeta_{i}^{\dagger} J\left(\Sigma \zeta_{i}^{\#}\right)=0$. Due to the semisimplicity hypothesis and (10b), different eigenspaces are $J$-orthogonal to each other as well, so that $\zeta_{i}^{\dagger} J \zeta_{j}=\left(\Sigma \zeta_{i}^{\#}\right)^{\dagger} J\left(\Sigma \zeta_{j}^{\#}\right)=\left(\Sigma \zeta_{i}^{\#}\right)^{\dagger} J \zeta_{j}=0$ for $i \neq j=1, \ldots, r_{+}$. If we define the matrix $\zeta \doteq\left[\zeta_{1} \ldots \zeta_{r_{+}}\right] \in \mathbb{C}^{2 n \times r_{+}}$, we see that

$$
\begin{aligned}
\mathcal{C}\left[\zeta \Sigma \zeta^{\#}\right]= & {\left[\zeta \Sigma \zeta^{\#}\right] \operatorname{diag}\left(\lambda_{1}^{+}, \ldots, \lambda_{r_{+}}^{+}, \lambda_{1}^{+}, \ldots, \lambda_{r_{+}}^{+}\right), } \\
& {\left[\zeta \Sigma \zeta^{\#}\right]^{\dagger} J\left[\zeta \Sigma \zeta^{\#}\right]=J_{2 r_{+} .} }
\end{aligned}
$$

The treatment of the negative eigenvalues is identical. The resulting matrix $\eta \in$ $\mathbb{C}^{2 n \times r_{-}}$satisfies the analogous relations

$$
\begin{aligned}
\mathcal{C}\left[\eta \Sigma \eta^{\#}\right]= & {\left[\eta \Sigma \eta^{\#}\right] \operatorname{diag}\left(\lambda_{1}^{-}, \ldots, \lambda_{r_{-}}^{-}, \lambda_{1}^{-}, \ldots, \lambda_{r_{-}}^{-}\right), } \\
& {\left[\eta \Sigma \eta^{\#}\right]^{\dagger} J\left[\eta \Sigma \eta^{\#}\right]=J_{2 r_{-}} . }
\end{aligned}
$$

Similarly, for the case of zero eigenvalues, the corresponding matrix $\psi \in \mathbb{C}^{2 n \times r_{0}}$ satisfies the relations

$$
\begin{gathered}
\mathcal{C}\left[\psi \Sigma \psi^{\#}\right]=0_{2 n \times 2 r_{0},} \\
C\left[\psi \Sigma \psi^{\#}\right]=0_{2 m \times 2 r_{0}}, \\
{\left[\psi \Sigma \psi^{\#}\right]^{\dagger} J\left[\psi \Sigma \psi^{\#}\right]=J_{2 r_{0}} .}
\end{gathered}
$$

The middle equation holds due to the fact $\operatorname{Ker} \mathcal{C}=\operatorname{Ker} C$; see Theorem 4.1. Finally, we consider the nonreal eigenvalues of $\mathcal{C}$ (with positive imaginary part), $\lambda_{i}^{c}=\mu_{i}+\imath \nu_{i}$, 
with $\nu_{i}>0, i=1 \ldots, r_{c}$. By Lemmas 4.2 and 4.3, there correspond four associated eigenvectors, $\xi_{i 1}, \xi_{i 2}, \Sigma \xi_{i 2}^{\#}$, and $\Sigma \xi_{i 1}^{\#}$, to each eigenvalue, where

$$
\begin{array}{ll}
\mathcal{C} \xi_{i 1}=\lambda_{i}^{c} \xi_{i 1}, & \mathcal{C}\left(\Sigma \xi_{i 2}^{\#}\right)=\lambda_{i}^{c}\left(\Sigma \xi_{i 2}^{\#}\right), \\
\mathcal{C} \xi_{i 2}=\lambda_{i}^{c *} \xi_{i 2}, & \mathcal{C}\left(\Sigma \xi_{i 1}^{\#}\right)=\lambda_{i}^{c *}\left(\Sigma \xi_{i 1}^{\#}\right),
\end{array}
$$

and

$$
\begin{aligned}
\xi_{i \alpha}^{\dagger} J \xi_{i \beta}^{\#} & =1-\delta_{\alpha \beta}, \\
\left(\Sigma \xi_{i \alpha}^{\#}\right)^{\dagger} J\left(\Sigma \xi_{i \beta}^{\#}\right) & =-1+\delta_{\alpha \beta}, \\
\left(\Sigma \xi_{i \alpha}^{\#}\right)^{\dagger} J \xi_{i \beta}^{\#} & =0 \quad \text { for } \alpha, \beta=1,2 .
\end{aligned}
$$

For our purposes, it will be beneficial to work with the linear combinations $\chi_{i 1} \doteq$ $\frac{1}{\sqrt{2}}\left(\xi_{i 1}+\xi_{i 2}\right)$ and $\chi_{i 2} \doteq \frac{1}{\sqrt{2}}\left(\xi_{i 1}-\xi_{i 2}\right)$, along with $\Sigma \chi_{i 1}^{\#}$ and $\Sigma \chi_{i 2}^{\#}$. It is straightforward to show that

$$
\mathcal{C}\left[\chi_{i 1} \Sigma \chi_{i 2}^{\#} \Sigma \chi_{i 1}^{\#} \chi_{i 2}\right]=\left[\chi_{i 1} \Sigma \chi_{i 2}^{\#} \Sigma \chi_{i 1}^{\#} \chi_{i 2}\right]\left(\begin{array}{rr}
\mu_{i} I_{2} & -\nu_{i} \sigma_{2} \\
\nu_{i} \sigma_{2} & \mu_{i} I_{2}
\end{array}\right)
$$

and

$$
\begin{array}{lrl}
\chi_{i 1}^{\dagger} J \chi_{i 1} & =\left(\Sigma \chi_{i 2}^{\#}\right)^{\dagger} J\left(\Sigma \chi_{i 2}^{\#}\right)=1, & \chi_{i 1}^{\dagger} J \chi_{i 2}=\left(\Sigma \chi_{i 1}^{\#}\right)^{\dagger} J\left(\Sigma \chi_{i 2}^{\#}\right)=0, \\
\chi_{i 2}^{\dagger} J \chi_{i 2}=\left(\Sigma \chi_{i 1}^{\#}\right)^{\dagger} J\left(\Sigma \chi_{i 1}^{\#}\right)=-1, & \left(\Sigma \chi_{i \alpha}^{\#}\right)^{\dagger} J \chi_{i \beta}=0 \quad \text { for } \alpha, \beta=1,2 .
\end{array}
$$

If we define $\chi \doteq\left[\chi_{11} \Sigma \chi_{12}^{\#} \ldots \chi_{r_{c} 1} \Sigma \chi_{r_{c} 2}^{\#}\right]$ and recall that different eigenvalue blocks are $J$-orthogonal to each other, we can see that the following relations hold:

$$
\begin{gathered}
\mathcal{C}\left[\chi \Sigma \chi^{\#}\right]=\left[\chi \Sigma \chi^{\#}\right]\left(\begin{array}{rr}
\mu & -\nu \\
\nu & \mu
\end{array}\right), \\
{\left[\chi \Sigma \chi^{\#}\right]^{\dagger} J\left[\chi \Sigma \chi^{\#}\right]=J_{4 r_{c}},}
\end{gathered}
$$

where $\mu=\operatorname{diag}\left(\mu_{1} I_{2}, \ldots, \mu_{r_{c}} I_{2}\right)$ and $\nu=\operatorname{diag}\left(\nu_{1} \sigma_{2}, \ldots, \nu_{r_{c}} \sigma_{2}\right)$.

Let us define $\bar{W} \doteq\left[[\zeta \eta \chi] \Sigma[\zeta \eta \chi]^{\#}\right]$ and $W \doteq\left[[\zeta \eta \chi \psi] \Sigma[\zeta \eta \chi \psi]^{\#}\right]$. Then, $W$ is Bogoliubov. Indeed, recalling the orthonormality relations within each type of eigenvector block (complex, real positive, real negative, and zero eigenvalues) and the fact that different blocks are $J$-orthogonal to each other, we can see that

$$
W^{\mathrm{b}} W=J\left(W^{\dagger} J W\right)=J J=I
$$

and

$$
\Sigma W \Sigma=\Sigma\left[\Sigma[\zeta \eta \chi \psi]^{\#}[\zeta \eta \chi \psi]\right]=\left[[\zeta \eta \chi \psi]^{\#} \Sigma[\zeta \eta \chi \psi]\right]=W^{\#} .
$$

We also have that

$$
\mathcal{C} \bar{W}=\bar{W} \overline{\mathcal{C}}=\bar{W}\left(\begin{array}{cc}
\overline{\mathcal{C}}_{1} & \overline{\mathcal{C}}_{2} \\
\overline{\mathcal{C}}_{2}^{\#} & \overline{\mathcal{C}}_{1}^{\#}
\end{array}\right)
$$

where

$$
\begin{aligned}
& \overline{\mathcal{C}}_{1}=\operatorname{diag}\left(\lambda_{1}^{+}, \ldots, \lambda_{r_{+}}^{+}, \lambda_{1}^{-}, \ldots, \lambda_{r_{-}}^{-}, \mu_{1} I_{2}, \ldots, \mu_{r_{c}} I_{2},\right), \\
& \overline{\mathcal{C}}_{2}=\operatorname{diag}(\underbrace{0, \ldots, 0}_{r_{+}+r_{-}},-\nu_{1} \sigma_{2}, \ldots,-\nu_{r_{c}} \sigma_{2}) .
\end{aligned}
$$

Copyright $@$ by SIAM. Unauthorized reproduction of this article is prohibited. 
$\overline{\mathcal{C}}$ is just the restriction of $\mathcal{C}$ on its $2 r$-dimensional invariant subspace spanned by eigenvectors with nontrivial eigenvalues $\left(r=r_{+}+r_{-}+2 r_{c}\right)$. We can factor $\overline{\mathcal{C}}=\bar{C}^{b} \bar{C}$, with $\bar{C} \doteq\left(\begin{array}{cc}\bar{C}_{1} & \bar{C}_{2} \\ \bar{C}_{2}^{\#} & \bar{C}_{1}^{\#}\end{array}\right) \in \mathbb{C}^{2 r \times 2 r}$, where

$$
\begin{aligned}
& \bar{C}_{1}=\operatorname{diag}(\sqrt{\lambda_{1}^{+}}, \ldots, \sqrt{\lambda_{r_{+}}^{+}}, \underbrace{0, \ldots, 0}_{r_{-}}, \alpha_{1} I_{2}, \ldots, \alpha_{r_{c}} I_{2}), \\
& \bar{C}_{2}=\operatorname{diag}(\underbrace{0, \ldots, 0}_{r_{+}}, \sqrt{\left|\lambda_{1}^{-}\right|}, \ldots, \sqrt{\left|\lambda_{r_{-}}^{-}\right|},-\beta_{1} \sigma_{2}, \ldots,-\beta_{r_{c}} \sigma_{2}) .
\end{aligned}
$$

The parameters $\alpha_{i}$ and $\beta_{i}$ are determined in terms of $\lambda_{i}^{c}$, as follows:

$$
\alpha_{i}=\sqrt{\frac{\left|\lambda_{i}^{c}\right|+\operatorname{re} \lambda_{i}^{c}}{2}}, \quad \beta_{i}=\frac{\operatorname{im} \lambda_{i}^{c}}{\sqrt{2\left(\left|\lambda_{i}^{c}\right|+\operatorname{re} \lambda_{i}^{c}\right)}} .
$$

Introducing the definition $\mathcal{C}=C^{b} C$ and the factorization $\overline{\mathcal{C}}=\bar{C}^{b} \bar{C}$ into (13), we compute

$$
C^{b} C \bar{W}=\bar{W} \bar{C}^{b} \bar{C} \Rightarrow \bar{W}^{b} C^{b} C \bar{W}=\bar{C}^{b} \bar{C} \Rightarrow\left(C \bar{W}(\bar{C})^{-1}\right)^{b} \cdot\left(C \bar{W}(\bar{C})^{-1}\right)=I .
$$

The fact that $\bar{C}$ is full rank was used in the above calculation to guarantee its invertibility. The matrix $C \bar{W}(\bar{C})^{-1} \in \mathbb{C}_{d u}^{2 m \times 2 r}$, since each of its factors has this property. Then, there exists a matrix $V_{I} \in \mathbb{C}^{2 m \times r}$, such that

$$
C \bar{W}(\bar{C})^{-1}=\left[\begin{array}{ll}
V_{I} & \Sigma V_{I}^{\#}
\end{array}\right] \Rightarrow C \bar{W}=\left[\begin{array}{ll}
V_{I} & \Sigma V_{I}^{\#}
\end{array}\right] \bar{C} .
$$

Notice that the columns of $\left[V_{I} \Sigma V_{I}^{\#}\right]$ are $J$-orthonormal, i.e., $\left[V_{I} \Sigma V_{I}^{\#}\right]^{\dagger} J\left[V_{I} \Sigma V_{I}^{\#}\right]=$ $J_{2 r}$. The final step is to complete a $J$-orthonormal basis of $\left(\mathbb{C}^{2 m}, J_{2 m}\right)$ with the doubled-up property, that is, to find a matrix $V_{I I} \in \mathbb{C}^{2 m \times(m-r)}$, such that $V \doteq$ $\left[\left[V_{I} V_{I I}\right] \Sigma\left[V_{I} V_{I I}\right]^{\#}\right]$ is Bogoliubov. To do this, consider the image of $\left[V_{I} \Sigma V_{I}^{\#}\right]$. It is a nondegenerate subspace of $\mathbb{C}^{2 m}$, meaning that it admits a $J$-orthonormal basis. Such a basis is, in fact, furnished by the columns of $\left[V_{I} \Sigma V_{I}^{\#}\right]$. It follows then [6] that its $J$-orthogonal complement in $\mathbb{C}^{2 m}$ is also nondegenerate; hence it also admits a $J$-orthonormal basis. Any such basis must contain $m-r$ vectors whose inner product with themselves is 1 , and $m-r$ vectors whose inner product with themselves is -1 . Then, $V_{I I}$ can be any matrix whose columns are composed of those basis vectors whose inner product with themselves is 1 . Finally, from (14) and $C\left[\psi \Sigma \psi^{\#}\right]=0$, we obtain

$$
\begin{gathered}
C\left[[\bar{W} \psi] \Sigma\left[\bar{W} \psi^{\#}\right]\right]=\left[\left[V_{I} V_{I I}\right] \Sigma\left[V_{I} V_{I I}\right]^{\#}\right]\left(\begin{array}{cc}
\left(\begin{array}{cc}
\bar{C}_{1} & 0 \\
0 & 0 \\
\bar{C}_{2} & 0 \\
0 & \# \\
0 & 0
\end{array}\right)^{\#} & \left(\begin{array}{cc}
\bar{C}_{2} & 0 \\
0 & 0 \\
\left(\bar{C}_{1}\right. & 0 \\
0 & 0
\end{array}\right)^{\#}
\end{array}\right) \\
\Rightarrow C W=V \hat{C},
\end{gathered}
$$

which is exactly the statement of the theorem, given that $W$ is Bogoliubov.

Before we employ the theorem to prove the analogue of Theorem 3.1 in the case of a general LQSS, we make several remarks.

Remark 4.4. In order to prove the analogue of Theorem 3.1 in the case of a general LQSS, it is necessary that $V$ be Bogoliubov and $W$ be doubled-up.

Copyright $@$ by SIAM. Unauthorized reproduction of this article is prohibited. 
Remark 4.5. The theorem excludes the case of a $C$ such that $\mathcal{C}=C^{b} C$ has nonsemisimple eigenvalues. Though there is no fundamental issue in extending the result in that case, there does not seem to exist a simple form for the reduced form $\hat{C}$.

Remark 4.6. The theorem also excludes the case where $\operatorname{Ker} C$ is a strict subspace of $\operatorname{Ker} \mathcal{C}$. When $\operatorname{Ker} \mathcal{C}=\operatorname{Ker} C, C$ is called $J$-nondegenerate, and this is the generic situation for $C \in \mathbb{C}_{d u}^{2 m \times 2 n}$, with $m \leq n$. The proof is as follows: Let us define the map $\phi_{m, n}: \mathbb{C}_{d u}^{2 m \times 2 n} \rightarrow \mathbb{R}^{2 m \times 2 n}$ by $\phi_{m, n}(C) \doteq \Phi_{2 m} C \Phi_{2 n}^{-1} . \phi_{m, n}$ is an isomorphism, which implies that $\operatorname{Rank} C=\operatorname{Rank} \phi_{m, n}(C)$. It is well known that a $2 m \times 2 n$ real matrix, with $m \leq n$, will have rank equal to $2 m$, generically. Hence, it follows that the generic $C \in \mathbb{C}_{d u}^{2 m \times 2 n}$, with $m \leq n$, has rank equal to $2 m$. Then, $\operatorname{Rank} C^{b}=\operatorname{Rank} J_{2 n} C^{\dagger} J_{2 m}=$ $\operatorname{Rank} C^{\dagger}=2 m$. From this, $\operatorname{Rank} \mathcal{C} \leq \min \left(\operatorname{Rank} C, \operatorname{Rank} C^{b}\right)=2 m$. But, from Sylvester's rank inequality, we have that $\operatorname{Rank} \mathcal{C} \geq \operatorname{Rank} C+\operatorname{Rank} C^{b}-2 m=2 m$. Hence, $\operatorname{Rank} \mathcal{C}=2 m$, so $\operatorname{dim} \operatorname{Ker} \mathcal{C}=2 n-2 m=\operatorname{dim} \operatorname{Ker} C$, from which $\operatorname{Ker} \mathcal{C}=\operatorname{Ker} C$ follows. In the case $m \geq n$, we have that $\operatorname{Ker} C C^{b}=\operatorname{Ker} C^{b}$, generically. Then, one can prove Theorem 4.1 using $C^{b}$ in place of $C$.

Remark 4.7. For $X \in \mathbb{R}^{2 r \times 2 s}$, define its $\sharp$-adjoint $X^{\sharp}$ by $X^{\sharp}=-\mathbb{J}_{2 s} X^{\top} \mathbb{J}_{2 r}$. The $\sharp$ adjoint satisfies properties similar to those of the usual adjoint, namely $\left(x_{1} A+x_{2} B\right)^{\sharp}=$ $x_{1} A^{\sharp}+x_{2} B^{\sharp},(A B)^{\sharp}=B^{\sharp} A^{\sharp}$, and $\left(A^{\sharp}\right)^{\sharp}=A$. Using the definition of the map $\phi$ in the previous remark, we have that for $C \in \mathbb{C}^{2 r \times 2 s}, \phi_{r, s}(C)^{\sharp}=\phi_{s, r}\left(C^{b}\right)$. Then, given a Bogoliubov matrix $T \in \mathbb{C}_{d u}^{2 k \times 2 k}, S=\phi_{k, k}(T) \in \mathbb{R}^{2 k \times 2 k}$ satisfies $S^{\sharp}=\phi_{k, k}\left(T^{b}\right)=$ $\phi_{k, k}\left(T^{-1}\right)=\left(\phi_{k, k}(T)\right)^{-1}=S^{-1}$. Such a matrix $S$ is called real symplectic. The set of these matrices forms a noncompact Lie group, known as the real symplectic group, which is homomorphic to the Bogoliubov group. Given the isomorphisms $\phi$ between real and doubled-up and between symplectic and Bogoliubov matrices, respectively, Theorem 4.1 may be restated as follows.

Theorem 4.8. Let $X \in \mathbb{R}^{2 m \times 2 n}$ and $\mathcal{X} \doteq X^{\sharp} X$. We assume that all the eigenvalues of $\mathcal{X}$ are semisimple, and that $\operatorname{Ker} \mathcal{X}=\operatorname{Ker} X$. Let $\lambda_{i}^{+}>0, i=1, \ldots, r_{+}$, $\lambda_{i}^{-}<0, i=1, \ldots, r_{-}$, and $\lambda_{i}^{c}$, with $\operatorname{im} \lambda_{i}^{c}>0, i=1 \ldots, r_{c}$, be the nontrivial eigenvalues of $\mathcal{C}$ (along with $\lambda_{i}^{c *}$ ). Then, there exist symplectic matrices $V \in \mathbb{R}^{2 m \times 2 m}$ and $W \in \mathbb{R}^{2 n \times 2 n}$ and a matrix $\hat{X}=\left(\begin{array}{cc}\hat{X}_{1} & \hat{X}_{2} \\ \hat{X}_{2} & \hat{X}_{3}\end{array}\right) \in \mathbb{R}^{2 m \times 2 n}$, such that $X=V \hat{X} W^{\sharp}$, with $\hat{X}_{l}=\left(\begin{array}{cc}\bar{X}_{l} & 0 \\ 0 & 0\end{array}\right), \bar{X}_{l} \in \mathbb{R}^{r \times r}\left(r=r_{+}+r_{-}+2 r_{c} \leq \min \{n, m\}\right)$ for $l=1,2,3$, and

$$
\begin{aligned}
& \bar{X}_{1}=\operatorname{diag}\left(\sqrt{\lambda_{1}^{+}}, \ldots, \sqrt{\lambda_{r_{+}}^{+}}, \sqrt{\left|\lambda_{1}^{-}\right|}, \ldots, \sqrt{\left|\lambda_{r_{-}}^{-}\right|}, \alpha_{1} I_{2}, \ldots, \alpha_{r_{c}} I_{2}\right), \\
& \bar{X}_{2}=\operatorname{diag}(\underbrace{0, \ldots, 0}_{r_{+}^{+}+r_{-}}, \beta_{1} \mathbb{J}_{2}, \ldots, \beta_{r_{c}} \mathbb{J}_{2}), \\
& \bar{X}_{3}=\operatorname{diag}\left(\sqrt{\lambda_{1}^{+}}, \ldots, \sqrt{\lambda_{r_{+}}^{+}},-\sqrt{\left|\lambda_{1}^{-}\right|}, \ldots,-\sqrt{\left|\lambda_{r_{-}}^{-}\right|}, \alpha_{1} I_{2}, \ldots, \alpha_{r_{c}} I_{2}\right) .
\end{aligned}
$$

$\alpha_{i}$ and $\beta_{i}$ are calculated in terms of $\lambda_{i}^{c}, i=1 \ldots, r_{c}$, by

$$
\alpha_{i}=\sqrt{\frac{\left|\lambda_{i}^{c}\right|+\operatorname{re} \lambda_{i}^{c}}{2}} \quad \text { and } \quad \beta_{i}=\frac{\operatorname{im} \lambda_{i}^{c}}{\sqrt{2\left(\left|\lambda_{i}^{c}\right|+\operatorname{re} \lambda_{i}^{c}\right)}} .
$$

4.2. Main result. Let us refer to Figure 3. It represents a particular configuration, composed of a pre- and a postprocessing (general) static network and a collection of beam splitters and single-mode optical cavities in a feedback connection through a 
third static network. The beam splitters realize the unitary $\mathbb{J}_{2}=\left(\begin{array}{cc}0 & 1 \\ -1 & 0\end{array}\right)$. There are four types of cavities: Type I cavities have a single passive interconnection port, and Type IIp/IIa have an additional passive or active system port, respectively. Finally, Type III cavities have one passive and one active system port each, in addition to their passive interconnection port. In the following, we prove that this sort of configuration can realize the transfer function of an arbitrary passive LQSS for an appropriate choice of parameters.

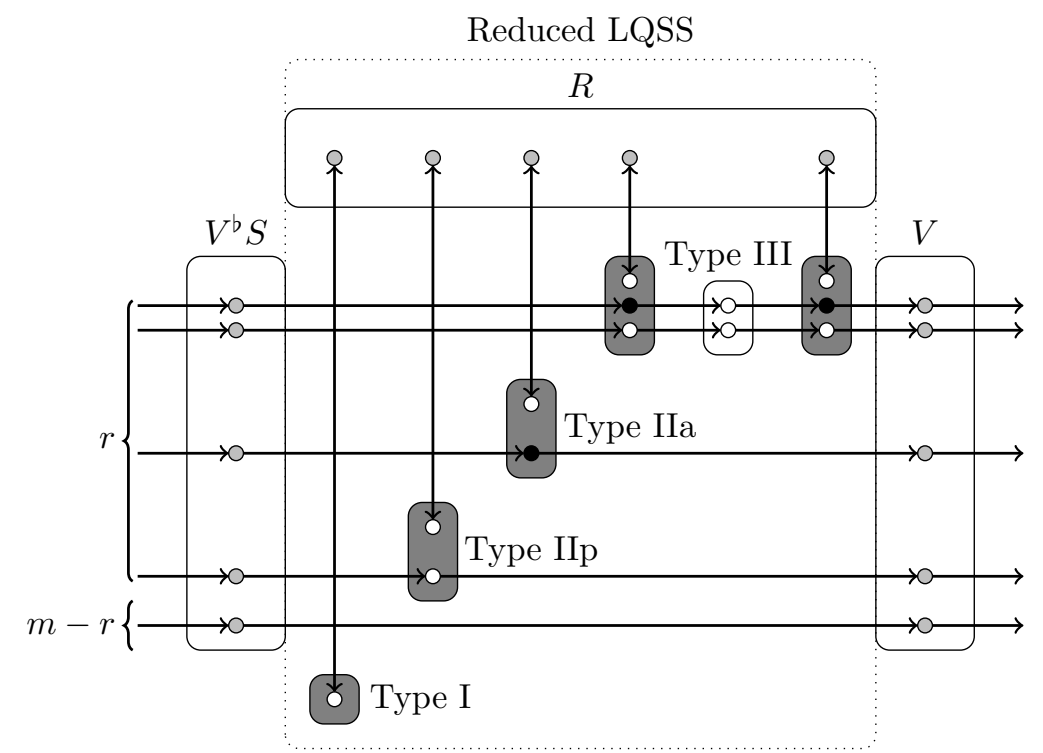

FIG. 3. A graphical representation of the realization of the transfer function of a general LQSS proposed in Theorem 4.9. Each cavity is representative of all cavities of its type.

THEOREM 4.9. Given a general LQSS with parameters $(S, C, \Omega)$, let

$$
G(s)=\left[I-C\left(s I+\imath J \Omega+\frac{1}{2} C^{b} C\right)^{-1} C^{b}\right] S
$$

be its transfer function. If $C$ satisfies the assumptions of Theorem 4.1, let $C=$ $V \hat{C} W^{b}$ be its corresponding decomposition. Then, $G(s)$ can be factorized as $G(s)=$ $V \hat{G}(s)\left(V^{b} S\right)$, where $\hat{G}(s)$ has the form

$$
\hat{G}(s)=I-\hat{C}\left(s I+\imath J \hat{\Omega}+\frac{1}{2} \hat{C}^{b} \hat{C}\right)^{-1} \hat{C}^{b},
$$

with $\hat{\Omega}=W^{\dagger} \Omega W . \hat{G}(s)$ is the transfer function of an LQSS with parameters $(I, \hat{C}, \hat{\Omega})$ and can be realized by the following feedback network of $n-r$ one-port, $r_{+}+r_{-}$ two-port, and $2 r_{c}$ three-port cavities, where $r=r_{+}+r_{-}+2 r_{c}$ :

$$
\begin{aligned}
d \check{a} & =\left(-\imath J \bar{\Omega}-\frac{1}{2} \tilde{C}^{b} \tilde{C}-\frac{1}{2} \hat{C}^{b} \hat{C}\right) \check{a} d t-\tilde{C}^{b} d \check{\mathcal{U}}_{i n t}-\hat{C}^{b} d \check{\mathcal{U}}, \\
d \check{\mathcal{Y}} & =\hat{C} \check{a} d t+d \check{\mathcal{U}} \\
d \check{\mathcal{Y}}_{i n t} & =\tilde{C} \check{a} d t+d \check{\mathcal{U}}_{i n t}, \\
d \check{\mathcal{U}}_{i n t} & =R d \check{\mathcal{Y}}_{i n t .} .
\end{aligned}
$$

Copyright (c) by SIAM. Unauthorized reproduction of this article is prohibited. 
Here, $\tilde{C}=\operatorname{diag}\left(\sqrt{\tilde{\kappa}_{1}}, \ldots, \sqrt{\tilde{\kappa}_{n}}, \sqrt{\tilde{\kappa}_{1}}, \ldots, \sqrt{\tilde{\kappa}_{n}}\right)$, and $\bar{\Omega}=\operatorname{diag}(D, D)+E+E^{\top}$, where

$$
D=\operatorname{diag}\left(\Delta_{1}^{+}, \ldots, \Delta_{r_{+}}^{+}, \Delta_{1}^{-}, \ldots, \Delta_{r_{-}}^{-}, \Delta_{1}^{c}, \Delta_{1}^{c}, \ldots, \Delta_{r_{c}}^{c}, \Delta_{r_{c}}^{c}, \Delta_{1}^{0}, \ldots, \Delta_{n-r}^{0}\right)
$$

and $E \in \mathbb{R}^{2 n \times 2 n}$ has all zero elements except for

$$
E_{r_{+}+r_{-}+2 i-1, n+r_{+}+r_{-}+2 i}=E_{r_{+}+r_{-}+2 i, n+r_{+}+r_{-}+2 i-1}=-\frac{\operatorname{im} \lambda_{i}^{c}}{2}
$$

for $i=1, \ldots, r_{c}$. The Bogoliubov matrix $R$ (feedback gain) is determined through the relations

$$
\begin{aligned}
X & =2 \imath\left(\tilde{C}^{b}\right)^{-1}(J \hat{\Omega}-J \bar{\Omega}) \tilde{C}^{-1}, \\
R & =(X-I)(X+I)^{-1} .
\end{aligned}
$$

Proof. The proof consists of two parts. First, we show that the LQSS

$$
\begin{aligned}
d \check{a} & =\left(-\imath J \bar{\Omega}-\frac{1}{2} \hat{C}^{b} \hat{C}\right) \check{a} d t-\hat{C}^{b} d \check{\mathcal{U}}, \\
d \check{\mathcal{Y}} & =\hat{C} \check{a} d t+d \check{\mathcal{U}}
\end{aligned}
$$

can be realized (in the strict sense) by a particular configuration of cavities. If we look at the structure of $\hat{C}$ and $\bar{\Omega}$, we conclude that it contains the following components:

1. $r_{+}$independent cavities with one passive port each, with Hamiltonian matrices $\operatorname{diag}\left(\Delta_{i}^{+}, \Delta_{i}^{+}\right)$and coupling matrices $\operatorname{diag}\left(\sqrt{\lambda_{i}^{+}}, \sqrt{\lambda_{i}^{+}}\right), i=1, \ldots, r_{+}$, corresponding to the positive eigenvalues of $\mathcal{C}$.

2. $r_{-}$independent cavities with one active port each, with Hamiltonian matrices $\operatorname{diag}\left(\Delta_{i}^{-}, \Delta_{i}^{-}\right)$and coupling matrices $\left(\begin{array}{cc}0 & \sqrt{\left|\lambda_{i}^{-}\right|} \\ \sqrt{\left|\lambda_{i}^{-}\right|} & 0\end{array}\right), i=1, \ldots, r_{-}$, corresponding to the negative eigenvalues of $\mathcal{C}$.

3. $r_{c}$ independent two-mode LQSSs with two ports, with Hamiltonian matrices

$$
\left(\begin{array}{cccc}
\Delta_{i}^{c} & 0 & 0 & -\operatorname{im} \lambda_{i}^{c} / 2 \\
0 & \Delta_{i}^{c} & -\operatorname{im} \lambda_{i}^{c} / 2 & 0 \\
0 & -\operatorname{im} \lambda_{i}^{c} / 2 & \Delta_{i}^{c} & 0 \\
-\operatorname{im~} \lambda_{i}^{c} / 2 & 0 & 0 & \Delta_{i}^{c}
\end{array}\right)
$$

and coupling matrices

$$
\left(\begin{array}{rrrr}
\alpha_{i} & 0 & 0 & \imath \beta_{i} \\
0 & \alpha_{i} & -\imath \beta_{i} & 0 \\
0 & -\imath \beta_{i} & \alpha_{i} & 0 \\
\imath \beta_{i} & 0 & 0 & \alpha_{i}
\end{array}\right)
$$

for $i=1, \ldots, r_{c}$, corresponding to the nonreal eigenvalues of $\mathcal{C}$. This LQSS can be realized as a cascade connection of two identical two-port, single-mode cavities and a beam splitter, as in Figure 4. Each cavity has one passive port with coupling coefficient $\alpha_{i}^{2}$ and one active port with coupling coefficient $\beta_{i}^{2}$. Its coupling matrix is given by

$$
\left(\begin{array}{rr}
0 & \imath \beta_{i} \\
\alpha_{i} & 0 \\
-\imath \beta_{i} & 0 \\
0 & \alpha_{i}
\end{array}\right)
$$

Copyright (c) by SIAM. Unauthorized reproduction of this article is prohibited. 


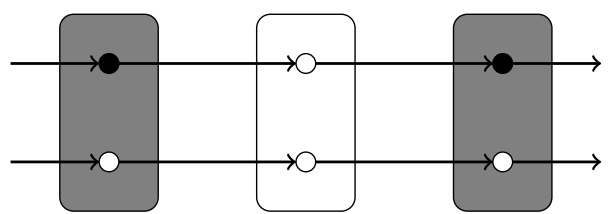

FIG. 4. A cascade system realizing the two-mode, two-port LQSS in the case of a complex eigenvalue.

and its Hamiltonian matrix is given by $\operatorname{diag}\left(\Delta_{i}^{c}, \Delta_{i}^{c}\right)$ for $i=1, \ldots, r_{c}$. The beam splitter implements the unitary transformation $\mathbb{J}_{2}=\left(\begin{array}{cc}0 & 1 \\ -1 & 0\end{array}\right)$.

4. $n-r$ closed cavities (without ports, that is), with Hamiltonian matrices $\operatorname{diag}\left(\Delta_{i}^{0}, \Delta_{i}^{0}\right)$ and coupling matrices equal to zero, $i=1, \ldots, r_{0}$, corresponding to the zero eigenvalues of $\mathcal{C}$.

Going from the configuration described by (17a) and (17b) to the reduced model described by (15a)-(15d) (see also Figure 3), we introduce a (passive) interconnection port in each cavity. To complete the proof, it suffices to prove that $\hat{G}(s)$ is the transfer function of the feedback network described by (15a)-(15d). To this end, we combine $(15 \mathrm{c})$ and $(15 \mathrm{~d})$ to obtain the relation $d \check{\mathcal{U}}_{i n t}=(I-R)^{-1} R \tilde{C} \check{a} d t$. Now, we introduce the Cayley transform $X=(I-R)^{-1}(I+R)$; see (8). It is straightforward to verify that $X$ is doubled-up and b-skew-Hermitian $\left(X^{\mathrm{b}}=-X\right)$ if and only if $R$ is Bogoliubov. The unique solution for $R$ in terms of $X$ is given by $R=(X-I)(X+I)^{-1}$. Using the identity $(I-R)^{-1} R=-\frac{1}{2} I+\frac{1}{2} X$, the relation between $d \check{\mathcal{U}}_{\text {int }}$ and $\check{a}$, and the definition of $X$, the equations for the reduced LQSS take the following form:

$$
\begin{aligned}
d \check{a} & =\left(-\imath J \bar{\Omega}-\frac{1}{2} \tilde{C}^{b} X \tilde{C}-\frac{1}{2} \hat{C}^{b} \hat{C}\right) \check{a} d t-\hat{C}^{b} d \check{\mathcal{U}}, \\
d \check{\mathcal{Y}} & =\hat{C} \check{a} d t+d \check{\mathcal{U}} .
\end{aligned}
$$

These equations describe an LQSS with Hamiltonian matrix $\hat{\Omega}$ given by the expression

$$
J \hat{\Omega}=J \bar{\Omega}-\frac{\imath}{2}\left(\tilde{C}^{b} X \tilde{C}\right) .
$$

Given any values for the cavity parameters $\Delta$ and $\tilde{\kappa}$ and any desired Hamiltonian matrix $\hat{\Omega}=W^{\dagger} \Omega W$, we may determine the unique $X$ (and, hence, the unique $R$ ) that produces this $\hat{\Omega}$ by the expression

$$
X=2 \imath\left(\tilde{C}^{b}\right)^{-1}(J \hat{\Omega}-J \bar{\Omega}) \tilde{C}^{-1} .
$$

Remark 4.10. Similarly to the passive case (see Remark 3.2) if $R=\left(\begin{array}{ll}R_{1} & R_{2} \\ R_{2}^{\#} & R_{1}^{\#}\end{array}\right)$, with $R_{1}$ and $R_{2}$ block-diagonal (with blocks of dimensions $r$ and $n-r$, respectively), the $n-r$ modes that are not influenced directly by the inputs are uncontrollable and unobservable. However, the controllability and observability properties of a general LQSS are more complicated [40].

4.3. Illustrative example. Consider a two-mode, two-port LQSS with the following parameters:

$$
\Omega=\left(\begin{array}{rrrr}
2 & 1 & 0 & -1 \\
1 & 2 & -1 & 0 \\
0 & -1 & 2 & 1 \\
-1 & 0 & 1 & 2
\end{array}\right), \quad C=\left(\begin{array}{rrrr}
0 & 1 & 2 & 0 \\
-1 & 2 & 1 & -1 \\
2 & 0 & 0 & 1 \\
1 & -1 & -1 & 2
\end{array}\right), \quad \text { and } S=I_{4}
$$


The eigenvalue decomposition of $\mathcal{C}=C^{b} C$ is computed to be $\mathcal{C}=U D U^{-1}$, where

$$
\begin{aligned}
D & =\operatorname{diag}(-2.8284,2.8284,-2.8284,2.8284), \\
U & =\left(\begin{array}{rrrr}
-0.9074 & 0.3474 & 0.2038 & 0.1756 \\
-0.1329 & 0.2965 & 0.4090 & -0.8908 \\
0 & 0 & -0.8629 & 0.4064 \\
0.3987 & -0.8896 & -0.2159 & -0.1027
\end{array}\right) .
\end{aligned}
$$

To the positive eigenvalue $\lambda^{+}=2.8284$, there correspond the eigenvectors $u_{2}$ and $u_{4}$ given by the second and fourth columns of $U$. We have that $\left\langle u_{4}, u_{4}\right\rangle_{J}>0$, and after normalization $u_{4}$ becomes $\zeta_{1}=(0.2180,-1.1061,0.5046,-0.1275)^{\top}$. To the negative eigenvalue $\lambda^{-}=-2.8284$, there correspond the eigenvectors $u_{1}$ and $u_{3}$ given by the first and third columns of $U$. We have that $\left\langle u_{1}, u_{1}\right\rangle_{J}>0$, and after normalization $u_{1}$ becomes $\eta_{1}=(-1.0987,-0.1609,0,0.4827)^{\top}$. According to the proof of Theorem 4.1,

$$
W=\left[\left[\zeta_{1} \eta_{1}\right] \Sigma\left[\zeta_{1} \eta_{1}\right]^{\#}\right]=\left(\begin{array}{rrrr}
0.2180 & -1.0987 & 0.5046 & 0 \\
-1.1061 & -0.1609 & -0.1275 & 0.4827 \\
0.5046 & 0 & 0.2180 & -1.0987 \\
-0.1275 & 0.4827 & -1.1061 & -0.1609
\end{array}\right)
$$

Since there are no zero eigenvalues,

$$
\hat{C}=\bar{C}=\left(\begin{array}{rrrr}
1.6818 & 0 & 0 & 0 \\
0 & 0 & 0 & 1.6818 \\
0 & 0 & 1.6818 & 0 \\
0 & 1.6818 & 0 & 0
\end{array}\right)
$$

and we can compute $V$ simply by

$$
V=C W \hat{C}^{-1}=\left(\begin{array}{rrrr}
-0.0576 & -1.0196 & 0.1834 & -0.0957 \\
-1.0691 & 0.0164 & 0.3357 & 0.1749 \\
0.1834 & -0.0957 & -0.0576 & -1.0196 \\
0.3357 & 0.1749 & -1.0691 & 0.0164
\end{array}\right)
$$

The Hamiltonian of the reduced LQSS is equal to

$$
\hat{\Omega}=W^{\dagger} \Omega W=\left(\begin{array}{rrrr}
3.6444 & 1.0135 & 0.4429 & -3.3952 \\
1.0135 & 4.3462 & -3.3952 & -1.7249 \\
0.4429 & -3.3952 & 3.6444 & 1.0135 \\
-3.3952 & -1.7249 & 1.0135 & 4.3462
\end{array}\right)
$$

The reduced LQSS can be implemented by the use of two cavities, one with a passive port (corresponding to $\lambda^{+}$) and one with an active port (corresponding to $\lambda^{-}$). Choosing the detuning of both cavities to be zero makes the total Hamiltonian of their concatenation $\Omega_{c o n c}=0_{4 \times 4}$. Also, we choose $\tilde{C}=I_{4}$. Then, we compute

$$
X=\imath\left(\begin{array}{rrrr}
7.2889 & 2.0271 & 0.8858 & -6.7904 \\
2.0271 & 8.6924 & -6.7904 & -3.4497 \\
-0.8858 & 6.7904 & -7.2889 & -2.0271 \\
6.7904 & 3.4497 & -2.0271 & -8.6924
\end{array}\right)
$$

from which the feedback gain $R$ is computed to be

Copyright $@$ by SIAM. Unauthorized reproduction of this article is prohibited. 


$$
R=\left(\begin{array}{rrrr}
-0.3731+7.8624 \imath & 0.9082-5.2659 \imath & 7.4743 \imath & 0.0450-5.8003 \imath \\
0.9082-5.2659 \imath & 0.3125+4.4401 \imath & -0.0450-5.8003 \imath & 3.7042 \imath \\
-7.4743 \imath & 0.0450+5.8003 \imath & -0.3731-7.8624 \imath & 0.9082+5.2659 \imath \\
-0.0450+5.8003 \imath & -3.7042 \imath & 0.9082+5.2659 \imath & 0.3125-4.4401 \imath
\end{array}\right)
$$

\section{REFERENCES}

[1] S. L. Braunstein, Squeezing as an irreducible resource, Phys. Rev. A, 71 (2005), 055801.

[2] O. Crisafulli, N. Tezak, D. B. S. Soh, M. A. Armen, and H. Mabuchi, Squeezed light in an optical parametric oscillator network with coherent feedback quantum control, Opt. Express, 21 (2013), pp. 18371-18386.

[3] S. C. Edwards and V. P. Belavkin, Optimal Quantum Filtering and Quantum Feedback Control, preprint, https://arxiv.org/abs/quant-ph/0506018, 2005.

[4] C. W. Gardiner and M. J. Collett, Input and output in damped quantum systems: Quantum stochastic differential equations and the master equation, Phys. Rev. A, 31 (1985), pp. 3761-3774.

[5] C. W. Gardiner and P. Zoller, Quantum Noise, 2nd ed., Springer-Verlag, Berlin, 2000.

[6] I. Gohberg, P. Lancaster, and L. Rodman, Matrices and Indefinite Scalar Products, Oper. Theory Adv. Appl. 8, Birkhäuser Verlag, Basel, 1983.

[7] G. H. Golub and C. F. V. Loan, Matrix Computations, 3rd ed., The Johns Hopkins University Press, Baltimore, MD, 1996.

[8] J. E. Gough, R. Gohm, and M. Yanagisawa, Linear quantum feedback networks, Phys. Rev. A, 78 (2008), 062104

[9] J. E. Gough AND M. R. James, The series product and its application to quantum feedforward and feedback networks, IEEE Trans. Automat. Control, 54 (2009), pp. 2530-2544.

[10] J. E. Gough, M. R. James, And H. I. Nurdin, Squeezing components in linear quantum feedback networks, Phys. Rev. A, 81 (2010), 023804.

[11] S. Grivopoulos, H. I. Nurdin, And I. R. Petersen, On transfer function realizations for linear quantum stochastic systems, in Proceedings of the 55th IEEE Conference on Decision and Control, 2016.

[12] R. Hamerly and H. Mabuchi, Advantages of coherent feedback for cooling quantum oscillators, Phys. Rev. Lett., 109 (2012), 173602.

[13] R. L. Hudson And K. R. Parthasarathy, Quantum Itô's formula and stochastic evolutions, Comm. Math. Phys., 93 (1984), pp. 301-323.

[14] M. R. James, H. I. Nurdin, and I. Petersen, $H^{\infty}$ control of linear quantum stochastic systems, IEEE Trans. Automat. Control, 53 (2008), pp. 1787-1803.

[15] E. Knill, R. Laflamme, and G. J. Milburn, A scheme for efficient quantum computation with linear optics, Nature, 409 (2001), pp. 46-52.

[16] K. Koga and N. Yамамото, Dissipation-induced pure Gaussian state, Phys. Rev. A, 85 (2012), 022103.

[17] U. Leonhardt, Quantum physics of simple optical instruments, Rep. Progr. Phys., 66 (2003), pp. 1207-1249.

[18] U. Leonhardt and A. Neumaier, Explicit effective Hamiltonians for general linear quantumoptical networks, J. Opt. B Quantum Semiclass. Opt., 6 (2004), pp. L1-L4.

[19] S. Ma, M. J. Woolley, I. R. Petersen, and N. Yamamoto, Preparation of pure Gaussian states via cascaded quantum systems, in Proceedings of the 2014 IEEE Conference on Control Applications, 2014.

[20] A. I. Maalouf and I. R. Petersen, Coherent $H^{\infty}$ control for a class of annihilation operator linear quantum systems, IEEE Trans. Automat. Control, 56 (2011), pp. 309-319.

[21] H. MabuChI, Coherent-feedback quantum control with a dynamic compensator, Phys. Rev. A, 78 (2008), 032323

[22] P. A. Meyer, Quantum Probability for Probabilists, 2nd ed., Springer-Verlag, Berlin, 1995.

[23] M. A. Nielsen And I. L. ChuAng, Quantum Computation and Quantum Information, Cambridge University Press, Cambridge, UK, 2000.

[24] H. I. NuRDin, On synthesis of linear quantum stochastic systems by pure cascading, IEEE Trans. Automat. Control, 55 (2010), pp. 2439-2444.

[25] H. I. NuRDin, Synthesis of linear quantum stochastic systems via quantum feedback networks, IEEE Trans. Automat. Control, 55 (2010), pp. 1008-1013.

[26] H. I. Nurdin, S. Grivopoulos, and I. R. Petersen, The transfer function of generic linear quantum stochastic systems has a pure cascade realization, Automatica J. IFAC, 69 (2016), pp. 324-333.

Copyright (C) by SIAM. Unauthorized reproduction of this article is prohibited. 
[27] H. I. Nurdin, M. R. James, And A. C. Doherty, Network synthesis of linear dynamical quantum stochastic systems, SIAM J. Control Optim., 48 (2009), pp. 2686-2718, https: //doi.org/10.1137/080728652.

[28] H. I. Nurdin, M. R. James, And I. R. Petersen, Coherent quantum LQG control, Automatica J. IFAC, 45 (2009), pp. 1837-1846.

[29] K. R. Parthasarathy, An Introduction to Quantum Stochastic Calculus, Birkhäuser, Basel, 1992.

[30] I. R. PETERSEn, Cascade cavity realization for a class of complex transfer functions arising in coherent quantum feedback control, Automatica J. IFAC, 47 (2011), pp. 1757-1763.

[31] I. R. Petersen, Quantum linear systems theory, Open Autom. Control Syst. J., 8 (2016), pp. 67-93.

[32] T. C. RALPH, Quantum optical systems for the implementation of quantum information processing, Rep. Progr. Phys., 69 (2006), pp. 853-898.

[33] M. Reck, A. Zeilinger, H. J. Bernstein, and P. Bertani, Experimental realization of any discrete unitary operator, Phys. Rev. Lett., 73 (1994), pp. 58-61.

[34] A. J. Shaiju And I. R. Petersen, A frequency domain condition for the physical realizability of linear quantum systems, IEEE Trans. Automat. Control, 57 (2012), pp. 2033-2044.

[35] D. F. Walls and G. J. Milburn, Quantum Optics, 2nd ed., Springer-Verlag, Berlin, 2008.

[36] H. M. Wiseman and G. J. Milburn, Quantum Measurement and Control, Cambridge University Press, Cambridge, UK, 2010.

[37] M. Yanagisawa And H. Kimura, Transfer function approach to quantum control-Part I: Dynamics of quantum feedback systems, IEEE Trans. Automat. Control, 48 (2003), pp. 21072120.

[38] M. Yanagisawa And H. Kimura, Transfer function approach to quantum control-Part II: Control concepts and applications, IEEE Trans. Automat. Control, 48 (2003), pp. 21212132.

[39] G. Zhang, Analysis of quantum linear systems' response to multi-photon states, Automatica J. IFAC, 50 (2014), pp. 442-451.

[40] G. Zhang, S. Grivopoulos, I. R. Petersen, and J. E. Gough, The Kalman decomposition for linear quantum systems, IEEE Trans. Automat. Control, (2017), https://doi.org/10. 1109/TAC.2017.2713343.

[41] G. Zhang And M. R. James, On the response of quantum linear systems to single photon input fields, IEEE Trans. Automat. Control, 58 (2013), pp. 1221-1235.

Copyright $@$ by SIAM. Unauthorized reproduction of this article is prohibited. 